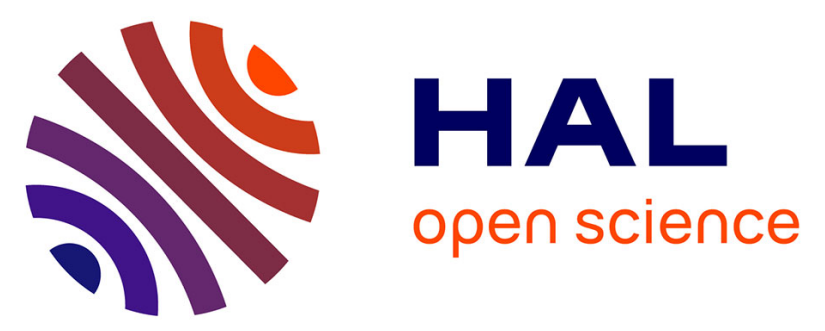

\title{
Skin layer stratification in drying droplets of dairy colloids
}

Ming Yu, Cécile Le Floch-Fouéré, Ludovic Pauchard, Françoise Boissel, Nan

$\mathrm{Fu}$, Xiao Dong Chen, Arnaud Saint-Jalmes, Romain Jeantet, Luca Lanotte

\section{- To cite this version:}

Ming Yu, Cécile Le Floch-Fouéré, Ludovic Pauchard, Françoise Boissel, Nan Fu, et al.. Skin layer stratification in drying droplets of dairy colloids. Colloids and Surfaces A: Physicochemical and Engineering Aspects, 2021, 620, pp.126560. 10.1016/j.colsurfa.2021.126560 . hal-03194114

\author{
HAL Id: hal-03194114 \\ https://hal.science/hal-03194114
}

Submitted on 22 Apr 2021

HAL is a multi-disciplinary open access archive for the deposit and dissemination of scientific research documents, whether they are published or not. The documents may come from teaching and research institutions in France or abroad, or from public or private research centers.
L'archive ouverte pluridisciplinaire HAL, est destinée au dépôt et à la diffusion de documents scientifiques de niveau recherche, publiés ou non, émanant des établissements d'enseignement et de recherche français ou étrangers, des laboratoires publics ou privés.

\section{(ㅇ)(1) $\$$}

Distributed under a Creative Commons Attribution - NonCommercial - NoDerivatives| 4.0 


\title{
Skin layer stratification in drying droplets of dairy colloids
}

Ming $\mathrm{Yu}^{\mathrm{a}}$, Cécile Le Floch-Fouéré ${ }^{\mathrm{a}}$, Ludovic Pauchard ${ }^{\mathrm{b}}$, Françoise Boissel ${ }^{\mathrm{a}}$, Nan Fu ${ }^{\mathrm{c}}$, Xiao Dong $\mathrm{Chen}^{\mathrm{c}}$, Arnaud Saint-Jalmes ${ }^{\mathrm{d}}$, Romain Jeantet ${ }^{\mathrm{a}}$, Luca Lanotte ${ }^{\mathrm{a}^{*}}$

a STLO, INRAE, Institut Agro 35042 Rennes (France)

${ }^{b}$ Université Paris-Saclay, CNRS, FAST, 91405, Orsay, France

${ }^{c}$ School of Chemical and Environmental Engineering, University of Soochow, 215000 Suzhou (China)

${ }^{d}$ Univ. Rennes, CNRS, IPR (Institut de Physique de Rennes) - UMR 6251, F-35000 Rennes, France

* Corresponding author - email address: luca.lanotte@inrae.fr

\begin{abstract}
The evaporation of a colloidal dispersion is characterized by solute accumulation at the air-liquid interface, leading to the gradual formation of a gelled skin. The development of this layer, from preliminary colloid deposit to complete solidification, affects overall drying process and final sample morphology. Despite, progress in the last decades, the mechanisms governing skin formation in drying colloidal suspensions have not yet been fully clarified, especially in complex polydisperse systems. In this work, we investigate this open question in droplets consisting of the two main milk proteins, i.e. whey proteins and casein micelles. Using complementary experimental approaches, we evaluate skin rheological behavior during the different stages of the evaporation process, highlighting the specific role of each colloid. Our results are interpreted in the light of drying-induced protein stratification, whose evidence is provided by the direct observation of dry skin section structure. This study contributes to the understanding of the competitive drying mechanisms occurring in binary colloidal systems. Moreover, our outcomes are potentially valuable for the optimization of milk powder production in dairy industry.
\end{abstract}

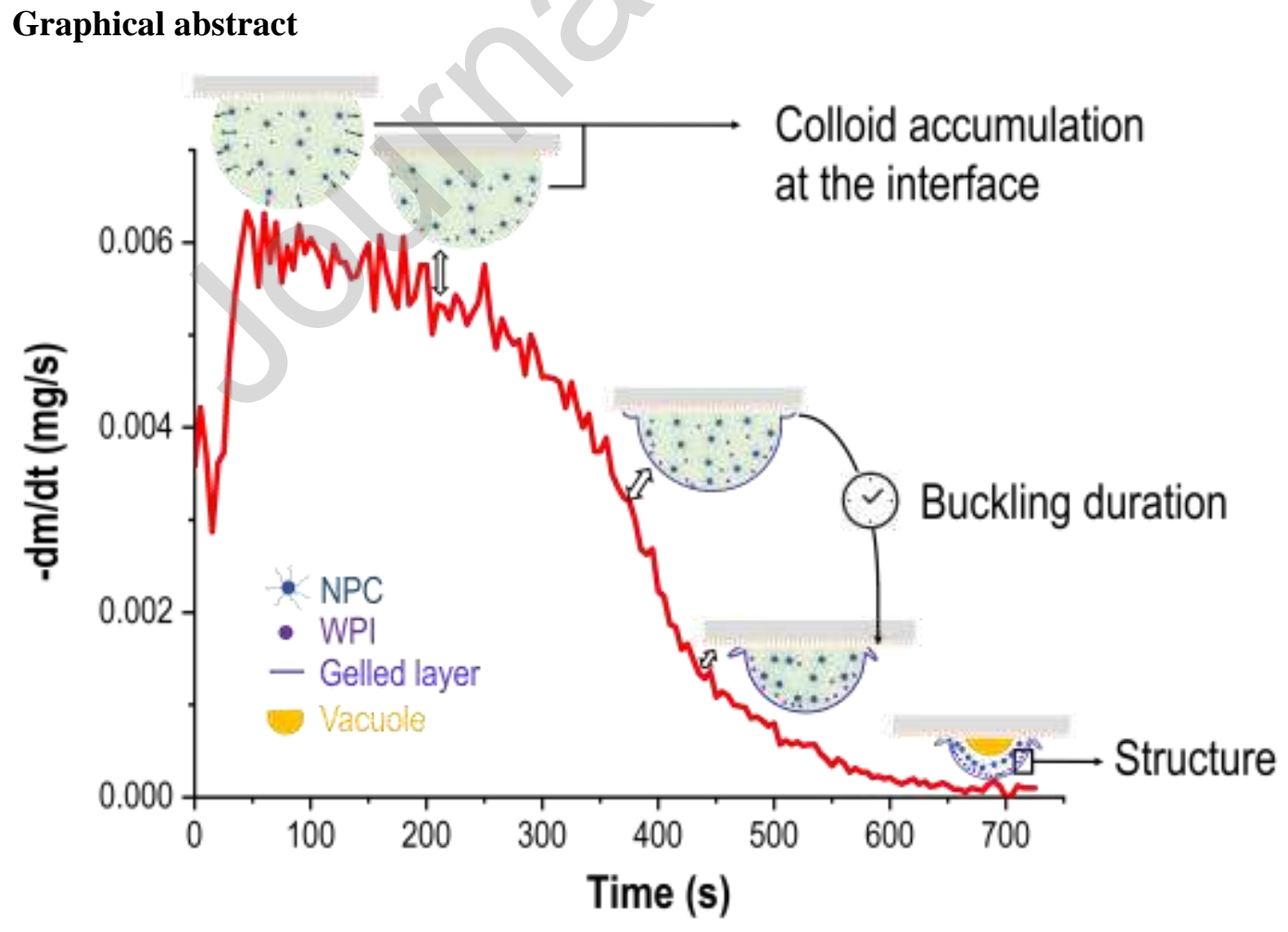




\section{Keywords: drying mechanisms, dairy colloids, skin formation, stratification}

\section{Introduction}

The effects of evaporation in colloidal systems are frequently observable in everyday life, starting from dry deposits of biological fluids (e.g. tears [1], saliva [2], and sweat [3,4]) to traces left by common beverages (e.g. coffee [5,6], tea [7,8]) on glass and other surfaces. Controlling the drying process in colloid dispersions is nowadays a challenge of great interest for the development of diagnostic techniques [9-11], the production of computer microchips [12], and the improvement of ink [13] and paint coatings [14]. Therefore, due to the numerous potential applications, this topic has been extensively studied in the last decades.

Most of the experimental works were performed on model colloids, well calibrated, to take advantage of the full characterization of their physico-chemical properties (e.g. latex and silica beads) [15]. This favored the investigation of the drying stages in different geometries, such as droplets [16-18], thin films [19], and confined setups (capillaries [20], Hele-Shaw cells [21,22]). Irrespective of the experimental approach, these studies recurrently highlighted gradual colloid accumulation at the air-liquid interface throughout the evaporation. In droplets, the migration of colloidal macromolecules towards the surface leads to the formation of a so-called skin, whose characteristics evolve from a gelled external layer to a final solid porous structure [18,23]. The skin development is essentially due to the competition between evaporation and diffusive Brownian motion. When the surface receding induced by evaporation overcomes the molecule diffusion, the colloids tend to locate at the surface and trigger the sol-gel transition once a solute critical concentration is locally achieved [24,25]. At the end of the evaporation process, the surface of dry samples can display different morphologies depending on solute properties and overall concentration. Indeed, the observation of highly deformed skin (i.e. buckling) [23,26], or conversely of rigid compact external shell (often at the origin of border delamination) [27,28], as well as the formation of cracks [28-30], can be considered as a "signature" of the dynamics occurring at the molecular scale. Thus, shedding light on drying-induced interface mechanisms in colloidal suspensions is clearly valuable to predict macroscopic sample characteristics, such as shape, mechanical response, and, in the case of biological samples, functional properties. At the same time, understanding the physics of skin formation still represents a scientific challenge, especially when investigating complex systems (e.g. biological fluids and industrial systems) whose multicomponents can differ by size, structure, and charge.

In the case of colloidal binary dispersions, the open question is to shed light on the impact of particle characteristics and interaction on deposit and re-arrangement in the vicinity of the air-liquid interface [31]. To this purpose, both experimental and theoretical works have been recently conducted on model particles differing merely by size [24,32-36]. As it concerns the drying of droplets, experiments performed on super hydrophobic substrates [37] or by acoustic levitation [38] suggested the enrichment of small particles on the external region of the shell by evaluating surface mechanical behavior. However, although quite innovative, these studies did not aim at exploring the possible mechanisms of segregation. In this regard, the work of Liu et al. [39] of 2019 is a precious exception. Here, the authors investigated the mechanisms governing the development of interface structure in drying droplets of bidisperse suspensions by experiments and simulations. Their outcomes revealed that surface self-organization, ranging from random packing to selective stratification of small colloids on the top layer, depends on experimental conditions (i.e. evaporation rate [40]) and solute properties (size, concentration). Similar scenarios have been observed in thin films, leading to the deduction of increasingly complex predictive models $[24,34,35,41]$ of which more accurate analysis will be provided in the following sections. In general, 
stratification by size has been associated with colloid cross-interactions driven by osmotic pressure $[24,41]$ and, more recently, to the motion in the direction opposite to the surface induced by local particle concentration gradients at the surface [42]. However, due to the multiple crucial parameters involved, these mechanisms have not been fully clarified and need further thorough experimental verification.

In this work, we investigated the evaporation process in pendant droplets consisting of a glaring example of biocolloids, i.e. two of the most represented proteins in milk: the whey proteins (in the form of whey protein isolates, WPI) and the caseins (in the form of micellar native phosphocaseinates, NPC). WPI macromolecules exhibit a globular shape (average size, $\mathrm{d}_{\mathrm{WPI}} \approx 10-20 \mathrm{~nm}$ ) [43], whereas NPC are typical sponge-like micelles $\left(\mathrm{d}_{\mathrm{NPC}}=100-300 \mathrm{~nm}\right)$ [44]. We focused on observing the main drying-induced morphological changes occurring in WPI/NPC mixes in order to extract a possible link between surface colloid organization at the molecular scale and skin rheology evolution. In the last years, our group carried out several works on the drying of WPI and NPC single protein dispersions, providing a full characterization of the main stages of the process and underlining the influence of protein properties on skin formation and final shape $[27,45,46]$. Indeed, the drying of rigid WPI colloids resulted in round hollow dry shells. On the other hand, the deformable nature of NPC colloids was reflected in the significant buckling of the surface. However, only preliminary studies have been performed on mixes of dairy proteins, inter alia revealing WPI over-representation on the top shell layer and, thus, possible stratification [47].

To verify this last hypothesis and its impact on droplet morphology, here we propose a systematic investigation of skin mechanical behavior during the evaporation. We first present a global overview of the drying process by droplet profile observation and mass measurements. Afterwards, we evaluate surface mechanical properties from shape characteristics and drying characteristic times as a function of the composition of the mixes. Mostly, we provide a direct observation of dry section structure highlighting the clues of possible protein segregation in the skin. We corroborate our findings in the light of previous works on bidisperse colloid dispersions, underlining the good agreement with theoretical models in the literature. Finally, we provide further evidence of preferential segregation in WPI/NPC mixes performing tests of interfacial rheology by the oscillatory drop method. Our outcomes contribute to improve the knowledge about the physics of the evaporation in binary colloidal dispersions. They also open the road to potential applications in the dairy industry, especially concerning the optimization and the control of the functional properties of infant milk formulas, of which WPI and NPC are main protein components.

\section{Materials and Methods}

\section{Sample preparation}

WPI and NPC suspensions with $8 \mathrm{wt} . \%$ total protein concentration were prepared by dissolving WPI and NPC commercial powders in deionized water containing $0.02 \mathrm{wt} . \%$ of sodium azide $\left(\mathrm{NaN}_{3}\right)$ as a bacteriostatic agent. WPI and NPC powders exhibited $86 \%$ and $82 \%$ protein content, respectively. The dispersions were stirred at room temperature $\left(\mathrm{T}=20^{\circ} \mathrm{C}\right)$ for $48 \mathrm{~h}$ to ensure full dissolution. The WPI/NPC mixtures were obtained successively by mixing the WPI and NPC pure suspensions and adjusting the final composition to obtain different WPI relative percentages WPI $\%_{\mathrm{R}}$ defined as:

$\mathrm{WPI} \%_{\mathrm{R}}=\frac{\mathrm{m}_{\mathrm{WPI}}}{\mathrm{m}_{\text {solute }}}$

Where $\mathrm{m}_{\text {solute }}$ refers to the total mass of WPI and NPC powders in the dispersions. WPI $\%_{\mathrm{R}}$ was hence adjusted to $0,20,50,80$, or $100 \%$. 


\section{Single droplet drying}

The single droplet approach was adopted to investigate the morphology evolution by profile view observation and, at a later stage, to estimate the drying kinetics by mass measurement.

The droplets of protein dispersions $\left(\mathrm{V}_{0}=0.5 \mu \mathrm{l}\right)$ were deposited in pendant configuration on a patterned substrate made of poly dimethyl siloxane (PDMS), as already described in previous works $[27,45]$. Afterwards, they were transferred into a sealed glass box equipped with zeolites (HG2-DES-3, Rot) to keep a low relative humidity $(\mathrm{RH} \approx 2 \%)$. The evaporation temperature was kept constant at $20 \pm 1^{\circ} \mathrm{C}$. A high-speed camera (Fastcam MC2 10,000 NB, Photron) was used to record droplet profile history (fps=0.2) during the evaporation process. A light source (Phlox 100/100 LLub) was placed in the opposite direction of the camera to get a uniform background. A custom image analysis software (ImageJ) was used to analyze the acquired image sequences. Experiments were repeated from 8 to 10 times for each sample.

The evaluation of the drying kinetics was carried out using a similar approach with respect to morphology observations. After being deposited on PDMS substrates, single droplets of WPI/NPC suspensions were gently deposited by tweezers in the weighing chamber of an ultra-micro balance (XP2U, Mettler Toledo), whose plate was previously surrounded by zeolites to control the relative humidity to $\approx 2 \%$. The droplet mass loss (accuracy of $0.1 \mu \mathrm{g}$ ) was measured at the same environmental conditions (T, RH) of droplet profile tests until a constant mass value was kept for $15 \mathrm{~s}$ (overall drying time, $\mathrm{t}_{\mathrm{dry}}$ ). Tests were repeated from 8 to 10 times for each sample, showing significant repeatability (absolute deviation around \pm 0.02 ).

\section{Scanning electron microscopy (SEM)}

Dry WPI/NPC droplets were observed by SEM (JEOL JSM 7100F) at $5 \mathrm{kV}$ to investigate the structure of the skin section as a function of WPI $\%_{\mathrm{R}}$.

\section{Interfacial adsorption and rheometry}

A pendant drop tensiometer (Tracker, Teclis-Scientific, France) was used to study the time evolution of the surface tension $(\gamma)$, and of the elastic and viscous moduli in WPI/NPC mixes with different WPI $\%_{\mathrm{R}}$. The preliminary measure of $\gamma$ allowed us to monitor the dynamics of adsorption, since the decrease of the surface tension with time reflected how fast and how much proteins got adsorbed at the interface. As the early stages of the adsorption occur within a timescale $(\approx 1 \mathrm{~s})$ too short for the apparatus, only the final portion of the phenomenon was explored. By analogy to typical results obtained for other concentrated protein dispersions, all the samples showed a similar behavior irrespective of WPI\% $\%_{\mathrm{R}}$. Indeed, the surface tensions reached their equilibrium values, which ranged between 48 and $49.8 \mathrm{mN} / \mathrm{m}$ (see Table 1), about 100 s after drop formation. By following the surface tension only, no more evolution was detected over time after this fast relaxation.

Once $\gamma$ equilibrium was reached, oscillatory experiments were performed to monitor interfacial viscoelasticity. WPI/NPC drops $\left(\mathrm{V}_{0}=8 \mu \mathrm{l}\right)$ were extruded from the tip of a syringe and subjected to controlled dilation/contraction oscillations (typical amplitude and period are given in the Results and Discussion section). From the observation of surface tension changes induced by drop area oscillation, we derived the time evolution of the complex viscoelastic modulus $(E)$, which consists of an elastic $\left(E^{I}\right)$ and a viscous part $\left(E^{I I}\right)$, and it is defined as:

$E=\sqrt{\left(E^{I}\right)^{2}+\left(E^{I I}\right)^{2}}$ 
Tests were performed at $\mathrm{T}=20^{\circ} \mathrm{C}$ and repeated at least 5 times for each sample, showing a good repeatability (average deviation lower than 10\%).

Table 1: Overall protein volume fraction $\left(\varphi_{\mathrm{p}}\right)$, WPI volume fraction $\left(\varphi_{\mathrm{WPI}}\right)$, and average surface tension $(\gamma)$ at the equilibrium in WPI/NPC droplets as a function of WPI\% $R$.

\begin{tabular}{|c|c|c|c|c|c|}
\hline $\mathbf{W P I} \%_{\mathbf{R}}$ & $0 \%$ & $20 \%$ & $50 \%$ & $80 \%$ & $100 \%$ \\
\hline $\boldsymbol{\varphi}_{\mathbf{p}}$ & 0.27 & 0.24 & 0.18 & 0.11 & 0.06 \\
\hline $\boldsymbol{\varphi}_{\mathbf{W P I}}$ & - & 0.01 & 0.03 & 0.05 & 0.06 \\
\hline $\boldsymbol{\gamma}(\mathbf{m N} / \mathbf{m})$ & $48.4 \pm 0.1$ & $48 \pm 0.5$ & $49 \pm 0.5$ & $48.6 \pm 0.4$ & $49.8 \pm 1$ \\
\hline
\end{tabular}

\section{Dimensionless numbers}

The Bond number, defined as

Bo $=\frac{\rho \cdot g \cdot \mathrm{R}_{0}^{2}}{\gamma}$

Where $\rho$ is droplet density, $g$ is gravity acceleration, $R_{0}$ is the initial droplet radius and $\gamma$ stands for droplet surface tension, provides an estimation of the relative impact of gravity and surface tension on droplet morphology. In the present study, Bo was calculated for all the samples at the beginning of the drying process, making it possible to estimate $\rho$ as the ratio of the initial mass of the droplet once deposited on the weighing plate of the ultra-micro balance and its initial volume, well controlled by a Hamilton microsyringe.

The Péclet number $(\mathrm{Pe})$ is a precious tool to characterize the relative contribution of advection and diffusion to colloid motion during the evaporation. The estimation of Pe for WPI ( $\left.\mathrm{Pe}_{\mathrm{WPI}}\right)$ and NPC $\left(\mathrm{Pe}_{\mathrm{NPC}}\right)$ was here carried out using the work of Liu et al. [41] on the drying of binary colloidal droplets as main reference. For spherical droplets, Pe is defined as the ratio between the characteristic times related to diffusion $\left(\tau_{\mathrm{d}}\right)$ and evaporation $\left(\tau_{\mathrm{ev}}\right)$. The characteristic diffusion time is equal to:

$\tau_{d}=\frac{R_{0}^{2}}{D_{p}}$

Where $\mathrm{D}_{\mathrm{p}}$ is the colloid diffusion coefficient. This parameter was calculated through the Stokes-Einstein relation, approximating WPI and NPC proteins to spherical particles at the early drying stage:

$\mathrm{D}_{\mathrm{p}}=\frac{k_{\mathrm{B}} \cdot \mathrm{T}}{3 \pi \eta \mathrm{d}}$

Here, $k_{\mathrm{B}} \mathrm{T}$ is the system thermal energy, $\eta$ is the suspension viscosity, and $\mathrm{d}$ is the average particle diameter, i.e. WPI $\left(\mathrm{d}_{\mathrm{WPI}}\right)$ and NPC $\left(\mathrm{d}_{\mathrm{NPC}}\right)$ average size in this case. The viscosity of WPI $\left(\eta_{\mathrm{WPI}} \approx 1.17^{*} 10^{-}\right.$ $\left.{ }^{3} \mathrm{~Pa}^{*} \mathrm{~s}\right)$ and NPC $\left(\eta_{\mathrm{NPC}} \approx 2.1 * 10^{-3} \mathrm{~Pa}^{*} \mathrm{~s}\right)$ dispersions was estimated by rheometry using Couette geometry. On the other hand, the evaporation characteristic time can be expressed as

$\tau_{\mathrm{ev}}=\frac{\mathrm{R}_{0}}{\mathrm{v}_{\mathrm{ev}}}$

Where $\mathrm{v}_{\mathrm{ev}}$ is the evaporation rate intended as droplet volume variation with time. This evaporation rate can be obtained by the expression: 
$\mathrm{v}_{\mathrm{ev}}=1 / 2(3 / 4 \pi)^{2 / 3}\left(a / \mathrm{R}_{0}\right)$

Where $a\left(\mu \mathrm{m}^{2} / \mathrm{s}\right)$ refers to the rate of surface reduction. The parameter $a$ has been determined by image analysis, evaluating the droplet volume variation with time, and also calculated using the equation proposed by Picknett and Bexon [48] for sessile droplets with spherical cap shape (not reported here for the sake of simplicity). In the latter case, we considered that the differences with our setup are geometrically negligible from the drying point of view. At the beginning of the drying process $(50 \mathrm{~s}<\mathrm{t}<100 \mathrm{~s})$, typical values for the surface reduction and the evaporation rate in WPI and NPC droplets were $a \approx 600 \mu \mathrm{m}^{2} / \mathrm{s}$ and $\mathrm{v}_{\mathrm{ev}} \approx 0.13 \mu \mathrm{m} / \mathrm{s}$, respectively. To summarize, the equation used here for Pe calculation at the early evaporation stage was:

$\mathrm{Pe}=\tau_{\mathrm{d}} / \tau_{\mathrm{ev}}=1 / 2(3 / 4 \pi)^{2 / 3}\left(a / \mathrm{D}_{\mathrm{p}}\right)$.

\section{Results and Discussion}

\section{Morphological evolution of WPI/NPC droplets}

A summary of the drying process in WPI/NPC mixes is presented in Figure 1, where the image sequences illustrate the morphological changes occurring in droplets with different WPI $\%_{R}$ throughout the evaporation. This qualitative overview allowed detecting the three main stages of the process, in agreement with previous works in the literature $[27,45,47]$.

1) At the beginning, all droplets showed similar contact angle $\left(\theta_{0} \approx 105^{\circ}\right)$ due to the hydrophobic properties of the PDMS support. The Bond number (Bo) was estimated $\approx 0.08$ for all mixes, thus excluding possible gravity-induced deformations. During the first drying stage (referred to as 1 in Figure 1), the droplets were characterized by the loss of the nearly spherical geometry and the gradual shrinkage induced by solvent evaporation. As it concerns the bulk, the initial protein volume fraction $\left(\varphi_{p}\right)$ was strongly depending on $\mathrm{WPI} \%_{\mathrm{R}}$. Taking into account WPI and NPC voluminosity, which is equal to $0.74 \mathrm{ml} / \mathrm{mg}$ [49] and $4.4 \mathrm{ml} / \mathrm{mg}$ [50] respectively, we estimated that $\varphi_{\mathrm{p}}$ ranged from a minimum of $\varphi_{\mathrm{WPI}}=0.06$ for WPI $\%_{\mathrm{R}}=100 \%$ to a maximum of $\varphi_{\mathrm{NPC}}=0.27$ for $\mathrm{WPI} \%_{\mathrm{R}}=0 \%$ (Table 1 ). Therefore, in this first part of the evaporation process, the suspensions were diluted enough to consider protein macromolecules as "hard spheres" with no significant interactions, except in the vicinity of the air-liquid interface [51]. Indeed, the progressive volume reduction possibly favored colloid accumulation at the air-liquid interface as a result of the competition between the receding of the evaporation front and diffusive motions. To verify such hypothesis, we calculated the Péclet number (Pe) at the initial conditions for both WPI and NPC and we obtained $\mathrm{Pe}_{\mathrm{WPI}}=6$ and $\mathrm{Pe}_{\mathrm{NPC}}=100$. This means that, under these experimental conditions, the evaporation prevails on diffusion and the formation of an interfacial colloidal layer is predictable.

2) The second stage of the drying process was marked by the evidence of sol-gel transition at droplet surface. First, the decrease of the contact angle $(\theta)$ induced by volume shrinkage led to the onset of capillary flows with increasing intensity once $\theta<90^{\circ}$. This kind of internal motion has been largely observed in colloidal dispersions (e.g. coffee ring effect) and results in the continuous transport of the solute towards droplet triple line, where the evaporation rate is maximal [16,52]. In the present work, the external segregation of WPI and NPC macromolecules resulted in the formation of a gelled "foot" conferring a sort of Mexican cap shape to the droplets (see first two columns of the stage 2 in Figure 1 for the different $\left.\mathrm{WPI} \%_{\mathrm{R}}\right)$. It is worth emphasizing that up to this point in the process all WPI/NPC droplets displayed qualitatively the same behavior irrespective of WPI $\%_{\mathrm{R}}$ and, mostly, no evidence of skin formation nor of its impact on the evaporation process was observed. It is only at the end of the Stage 2 , 
i.e. when the sol-gel transition was advanced, that the signs of surface gelation became glaring, resulting in two main morphology categories depending on $\mathrm{WPI} \%_{\mathrm{R}}$. For $\mathrm{WPI} \%_{\mathrm{R}} \leq 20 \%$, the droplets underwent a typical buckling process with slight diameter reduction, whereas, for $\mathrm{WPI} \%_{\mathrm{R}} \geq 50 \%$, they exhibited a round smooth surface and evident border detachment from the substrate (i.e. delamination).

3) During the last stage of the evaporation process (referred to as 3 in Figure 1), no shape modifications were observed. The evaporation of the remaining water did not affect the diversification into two shape categories mainly distinguished by buckling (blue frame) and convex smooth surface (red frame) respectively, and it was accompanied by the formation or the definitive development of a vacuole in the nearly solid droplets. The different appearance of the two morphology types is not an unusual outcome. In fact, wrinkled (e.g. NPC) and Mexican cap shapes (e.g. WPI) have been often observed in previous works on model colloidal systems and in biological samples, depending on solute concentration, solvent composition, and experimental conditions ( $\mathrm{T}, \mathrm{RH}$ ) [23]. Buckling phenomena are secondary level instabilities occurring when droplet skin does not withstand the increasing inward pressure developing during the drying. Therefore, our observations suggest that WPI physico-chemical properties can confer a higher rigidity to the interface even in the simultaneous presence of NPC. Such enhanced robustness could depend not exclusively on colloid properties, but also on skin structural characteristics. In a previous work, for example, Head suggests that dry shells with homogeneous thickness do not undergo buckling instabilities [53]. In this light, the outcomes of Sadek et al. [27] on the drying of WPI droplets experimentally corroborate this theory, since samples exhibited an almost constant thickness from the base to the apex height for different protein concentrations.

The qualitative picture presented in Figure 1 provides two points of reflection:

- The drying dynamics in WPI/NPC mixes are strongly affected by sample composition (WPI\% $\%_{\mathrm{R}}$ ), resulting in a shape transition occurring at a critical WPI relative concentration (WPI $\%_{\mathrm{Rc}}$ ) found between for $20 \%$ and $50 \%$. The marked differences between the two observed morphologies possibly suggest a competitive separate role played by the two components on the drying process.

- WPI colloids have a significant impact on evaporation process and interface rheological properties. Indeed, despite being always the minor component in the mixes in terms of volume fraction (e.g., in mixes with $\mathrm{WPI} \%_{\mathrm{R}}=50 \%, \varphi_{\mathrm{WPI}}=0.03$ compared to overall $\varphi_{\mathrm{p}}=0.18$ ), whey proteins govern droplet final shape if sufficiently represented in the system $\left(\mathrm{WPI} \%_{\mathrm{R}} \geq 50 \%\right)$.

Starting from these preliminary conclusions, it would be important to understand if the dynamics leading to skin formation in drying WPI/NPC droplets, and consequently the evolution of surface mechanical properties, merely depend on colloid concentration in the sample or if there is any further impact due to a specific organization of WPI and NPC at the air-liquid interface.

\section{Drying kinetics}

The evaporation rate was monitored in all WPI/NPC mixes to investigate the link between droplet morphology evolution and drying kinetics. In Figure 2, the mass loss with time (-dm/dt) is displayed for single protein suspensions, i.e. WPI $\left(\mathrm{WPI} \%_{\mathrm{R}}=100 \%\right)$ and $\mathrm{NPC}\left(\mathrm{WPI} \%_{\mathrm{R}}=0 \%\right)$, and for a mix with $\mathrm{WPI} \%_{\mathrm{R}}=50 \%$. Compared to profile visualization in Figure 1 , which is limited by the difficulty of detecting early evidence of skin gelation, the evaluation of the kinetics allowed a better characterization of the duration of the different steps of the drying process. After an initial instability following droplet deposition on the substrate ( $\leq 1 \mathrm{~min}$, light yellow rectangle), the Stage 1 , characterized by droplet shrinkage, here corresponded to a slightly decreasing evaporation rate with time. Afterwards, compatibly with the progress of the sol-gel transition at droplet surface, a typical falling drying rate was observed (Stage 2). At the end of this phase, when no significant shape changes were observed, the evaporation rate 
went rapidly to zero (Stage 3). Upon a first examination, the curves in Figure 2 point out, on the one hand, the evident difference between NPC and WPI behaviors and, on the other, a relative similarity between WPI suspensions and mixes with WPI $\%_{\mathrm{R}}=50 \%$. In fact, NPC droplets exhibited an earlier falling drying stage and a retarded achievement of the plateau at the end of the evaporation compared to the other samples. This behavior is further highlighted in the inset of Figure 2, where the overall drying time ( $t_{\text {dry }}$, black triangles, see the Materials and Methods section for the definition) and the half-life drying time ( $\mathrm{t}_{1 / 2}$, blue stars) are displayed as a function of $\mathrm{WPI} \%_{\mathrm{R}}$. NPC dispersions were characterized by lower $\mathrm{t}_{1 / 2}$ (i.e. earlier sol-gel transition) and longer $t_{\text {dry }}$ than WPI-rich samples (WPI $\%_{R} \geq 50 \%$ ). The earlier occurrence of surface gelation in NPC dispersions has been already experimentally documented in the literature, using both single droplet [45] and confined geometry approaches [46]. Such behavior resulted in a lower critical mass concentration required to trigger interfacial sol-gel transition in NPC (16wt.\%) than in WPI samples (41wt.\%) [27]. The origin of such evident difference can be plausibly attributed to three main factors: i) the higher $\varphi_{\mathrm{p}}$ in NPC dispersions, despite the same fixed mass concentration, ii) the NPC micellar open structure, which is a glaring example of biological microgel retaining water, and iii) $\mathrm{Pe}_{\mathrm{NPC}}>>\mathrm{Pe}_{\mathrm{WPI}}$. In a few words, the abundant presence of casein micelles in the bulk suspension and their physico-chemical and structural properties favor the accumulation at droplet air-liquid interface and the formation of a concentrated layer with high water affinity. If on the one hand, the significant propensity of NPC colloids to foster the formation of a gelled interface finds a solid explanation even in the light of previous works, on the other, the detection of a longer drying time in NPC droplets than in WPI ones may appear striking. Indeed, one could predict that, due to their rigid globular structure, WPI colloids contribute to the development of a compact skin, whereas irregular and deformable NPC micelles would self-organize in a sparse porous structure. Consequently, a highly packed WPI-rich skin, similar to the case of monodisperse colloidal spheres, should represent a higher "resistance" to water evaporation than a sparse NPC surface layer. However, this simplistic, but plausible, point of view neglects some fundamental mechanisms characterizing the interface evolution in NPC suspensions. In a series of works on the rheology of casein micelle dispersions under osmotic stress, Bouchoux et al. [44,50,51] showed that NPC phase characteristics significantly evolve with increasing concentrations. They pointed out that above a specific concentration, comparable to mentioned values related to sol-gel transition in droplets, NPCs organize in a highly packed structure (packing factor $\approx 0.78$ higher than in monodisperse hard-sphere fluids). Such a dense arrangement is mainly affected by the polydispersity of average NPC size. The further increase of sample concentration induced by higher osmotic pressures implicates also micelle squeezing and intermicellar bonds leading to the development of a compact gel. The scenario explored by Bouchoux et al. is consistent with the physical events occurring at droplet surface during the evaporation process, thus providing a credible interpretation to the longer $\mathrm{t}_{\mathrm{dry}}$ measured for NPC samples in Figure 2.

As regards the analogy of behavior in WPI/NPC mixes with $\mathrm{WPI} \%_{\mathrm{R}} \geq 50 \%$ (the WPI $\%_{\mathrm{R}}=80 \%$ curve is not illustrated here for the sake of clarity), in agreement with the outcomes of Figure 1, it is evident that the presence of WPI colloids represents a limiting factor in the drying process. The main open question to address lies in understanding how whey protein physico-chemical properties affect skin structure and mechanical response: is it sort of mechanical reinforcement effect due to WPI macromolecules randomly dispersed in the interfacial layer? Or is it related to some spatial colloid stratification? This latter phenomenon has been frequently observed in polydisperse colloidal systems and it consists of the selective segregation of the components into separate layers having specific rheological and mechanical roles. Numerous experimental studies, as effectively summarized by Schulz and Keddie in a review of 2018 [31], highlighted that the surface region is responsible for defining fundamental properties, such as wetting, hardness, and electrical conductivity. For example, Nunes et al. [54] detected greater mechanical resistance in auto-stratified colloidal coatings by enriching the surface in hard particles. In our binary system, WPI colloids can be approximated to hard spherical colloids. Moreover, above the threshold value 
WPI $\%_{\mathrm{Rc}}$ ranging between $20 \%$ and $50 \%$, they clearly governed shape transition and skin mechanical properties. Therefore, in the next sections of this work we aimed at exploring possible clues of WPI outer accumulation during the evaporation process.

\section{Sol-gel transition}

To investigate the shape changes in WPI/NPC droplets, we evaluated two main parameters during the evaporation: i) the base diameter, D (i.e. the average diameter of the surface adhering to the PDMS substrate), and ii) the apex height, $\mathrm{H}$ (i.e. the distance from droplet top to substrate). This approach was helpful to characterize sol-gel transition major morphological consequences [27,45,47]: skin buckling and border delamination. Such shape modifications occurred in the final part of the Stage 2 (Figure 1) and led to the attenuation of $\mathrm{H}$ curve slope and, at the same time, to the sudden $\mathrm{D}$ reduction (see the curves in the inset of Figure 3 concerning WPI droplets), with an importance depending on WPI $\%_{\mathrm{R}}$. Indeed, both buckling and delamination represent, in a different way, a reaction to the increasing drying-induced inward pressures and the selective occurrence of either events is related to skin mechanical properties. For $\mathrm{WPI} \%_{\mathrm{R}} \leq 20 \%$, the abundant NPC amount provided surface deformability, resulting in skin folding. On the other hand, the mixes with WPI $\%_{\mathrm{R}} \geq 50 \%$ exhibited such a skin hardness that they resisted to interfacial pressure until stress release by delamination. Here, we defined as $\Delta \mathrm{t}_{\mathrm{gel}}$ the temporal range corresponding to such significant morphological changes, as illustrated in the inset of Figure 3, and we measured its onset and duration as a function of WPI $\%_{\mathrm{R}}$ (purple rectangles in Figure 3, values reported in Table 2). The dispersions with hard skin and evident delamination (WPI $\%_{\mathrm{R}} \geq 50 \%$ ) exhibited almost identical $\Delta \mathrm{t}_{\text {gel }}$, thus suggesting a similar surface mechanical response to stress even when WPI colloids are minor components in terms of volume fraction. This would corroborate the hypothesis of whey protein accumulation on the external part of droplet surface. On the contrary, the droplets undergoing buckling (WPI\% $\%_{\mathrm{R}} \leq 20 \%$ ) displayed a longer $\Delta \mathrm{t}_{\text {gel }}$, denoting slower gelation and different rheological behavior. Surprisingly, $\Delta \mathrm{t}_{\text {gel }}$ related to mixes with $\mathrm{WPI} \%_{\mathrm{R}}=20 \%$ was even longer than in single protein NPC droplets probably due to the impact of the presence of both proteins at the air-liquid interface.

The upper limit of $\Delta t_{\text {gel }}$ corresponded to the achievement of droplet shape invariancy for all samples. However, when comparing this value to the overall drying time $\left(t_{\text {dry }}\right)$ showed in Figure 2 , it seems evident that the end of gelation events did not reflect the end of the evaporation process. It is likely to predict that, despite skin solidification, some water could be still retained in droplet surface structure. The duration of this further water evaporation (final drying, $\Delta \mathrm{t}_{\text {fdry }}$ ), which provides a quantitative characterization of the Stage 3 in Figure 1, was significantly increasing with the amount of casein micelles in the dispersion (see values in Table 2). In the case of $\mathrm{WPI} \%_{\mathrm{R}} \geq 50 \%$, on the one hand droplet shape and skin mechanical behavior suggested WPI segregation on the outer surface region. On the other, the gradual increase of $\Delta t_{\text {fdry }}$ with NPC concentration pointed out that casein micelles were, in a certain way, still wet at the end of the process and thus, possibly accumulated in the inner skin layer.

Table 2: Duration of $\Delta t_{\text {gel }}$ and of the final drying of remaining water $\left(\Delta t_{\text {fdry }}\right)$ as a function of WPI\% $\%_{R}$

\begin{tabular}{|c|c|c|c|c|c|}
\hline $\mathbf{W P I}_{\mathbf{R}}$ & 0 & 20 & 50 & 80 & 100 \\
\hline$\Delta \mathbf{t}_{\text {gel }}(\mathbf{s})$ & $156 \pm 27$ & $204 \pm 47$ & $114 \pm 47$ & $113 \pm 27$ & $107 \pm 8$ \\
\hline$\Delta \mathbf{t}_{\text {fdry }}(\mathbf{s})$ & $130 \pm 20$ & $100 \pm 39$ & $126 \pm 42$ & $76 \pm 15$ & $42 \pm 11$ \\
\hline
\end{tabular}

\section{Structure of dry droplet section}

The hypothesis of drying-induced WPI colloid external segregation was further investigated observing the section structure of WPI/NPC dry droplets by SEM. On average, all mixes exhibited similar skin thickness 
$(\approx 60 \mu \mathrm{m})$. Our window of visualization was reduced to the region in the vicinity of the air-liquid interface (white frame in Figure 4A). Starting from the qualitative observation of WPI (WPI $\%_{\mathrm{R}}=100 \%$ ) and NPC $\left(\mathrm{WPI} \%_{\mathrm{R}}=0 \%\right)$ peculiarities (e.g. smoothness, porosity), the goal was to address an eventual protein stratification in mixes with WPI $\%_{\mathrm{R}}=50 \%$. Typical WPI droplet dry sections (Figure 4B) displayed a dense, smooth structure, whereas NPC shells (Figure 4C) were characterized by compact regions and sparse empty cells especially in proximity to droplet surface. In fact, the almost monodisperse size and the globular rigid structure of WPI colloids led to the formation of a homogenous, dense skin, as normally observed in hard spheres colloidal dispersions. On the contrary, due to their high polydispersity, deformation and bond development, NPC micelles enhanced significant compaction, but also the formation of pores irregularly dispersed in the shell layer. In mixes with WPI $\%_{\mathrm{R}}=50 \%$, both WPI and NPC skin properties were distinguished depending on the distance from the surface (Figure 4D). Indeed, the top layer showed homogeneous compactness and porosity, similar to WPI, but the internal part of the shell exhibited NPC-like irregularities and cavities.

Considering that $\mathrm{Pe}_{\mathrm{NPC}}>>\mathrm{Pe}_{\mathrm{WPI}}>1$, we could predict a faster accumulation of NPC micelles at the air-liquid interface during the drying process. Consequently, preferential segregation of the larger colloid on surface top layer should be expected in the binary system investigated in this work. However, the analysis of droplet shape changes with time (Figure 1), of drying kinetics (Figure 2), of skin mechanical behavior (Figure 3) coupled to the direct observation of dry droplet skin structure (Figure 4) rather underlines the external segregation of WPI colloids. To shed light on the mechanisms of skin formation in drying WPI/NPC droplets, it is necessary to refer to the rich literature concerning the evaporation in films and droplets of binary colloidal systems. In this respect, numerous experimental and theoretical works have been carried out in the last decade, focusing mainly on the behavior of model colloids differing merely by size [31]. According to more recent outcomes, the stratification of the smaller particles on the external part of the skin layer (i.e. small-on-top) can take place when the evaporation rate is predominant on diffusive phenomena $(\mathrm{Pe}>1)$ for both colloids $[24,35,41,42]$, which corresponds to our experimental scenario. In fact, the concentration gradients developing in the direction of the surface during the evaporation result in increasing osmotic pressures, which in turn can induce downward motion especially in larger particles. Starting from this hypothesis and modeling the diffusive cross-interactions between colloids, Zhou, Jiang and Doi (ZJD) [41] identified three crucial parameters to predict a possible stratification by size: the size ratio $(\alpha)$, the Péclet number $\left(\mathrm{Pe}_{\mathrm{s}}\right)$, and the volume fraction $\left(\varphi_{\mathrm{s}}\right)$ associated to the smaller colloid. They also deduced that the necessary condition for the small-on-top stratification to occur is that

$$
\alpha^{2}\left(1+\mathrm{Pe}_{\mathrm{s}}\right) \varphi_{\mathrm{s}}>\mathrm{C},
$$

Where $C$ is a constant $\approx 1$. In our binary dairy protein system, $\alpha=\mathrm{d}_{\mathrm{NPC}} / \mathrm{d}_{\mathrm{WPI}}=10-30, \mathrm{Pe}_{\mathrm{s}}=\mathrm{Pe}_{\mathrm{WPI}}=6$, and $\varphi_{\mathrm{s}}=\varphi_{\mathrm{WPI}}=0.01-0.06$. Therefore, the ZJD criterion is satisfied for all the WPI/NPC mixes investigated in this work but for $\mathrm{WPI} \%_{\mathrm{R}}=20 \%$, where the outcome is on the same order of $\mathrm{C}$. This could mean that in this kind of mixes with largely predominant NPC micelles, under these specific environmental conditions, droplet skin does not exhibit evident self-organization, i.e. protein stratification could not take place or at least not in the expected way. Despite the ZJD model represents a powerful tool to predict skin structure in binary colloid systems, it neglects hydrodynamic interactions and fluid backflow during the drying process. Sear and Warren [42], for example, proposed a revised model taking into account diffusiophoretic particle motion driven by concentration gradients, which attenuate the impact of the already mentioned osmotic pressure and, thus, downward flows. All in all, considering diffusiophoresis does not exclude the occurrence of surface stratification, but it implies higher colloid concentrations and faster evaporation rates. However, full modeling colloidal selective segregation remains a current open question requiring further thorough experimental and theoretical testing. For this reason, the good agreement between our 
experimental results and the ZJD model corroborates already the hypothesis of WPI colloids on top of NPC micelles in droplet dry skin, at least for higher WPI concentrations (higher than WPI\% $\%_{\mathrm{Rc}}$, i.e. $0.01 \leq \varphi_{\mathrm{WPI}} \leq 0.03$, as suggested in previous sections of this work).

\section{Colloid adsorption at droplet interface and gelation over time}

In principle, phenomena of self-organization at the air-liquid interface in colloidal systems are not limited to the study of the evaporation. For example, surface gelation can be frequently observed during the investigation of protein adsorption due to gradual ageing. As in the case of drying-induced skin formation, the continuous protein accumulation at the interface leads to the development of concentration gradients and, consequently, of osmotic pressures and, possibly, diffusiophoretic motion. Therefore, the experimental conditions could be favorable for colloid stratification. In the case of WPI/NPC mixes examined in our study, it is interesting to check whether selective protein segregation induced by surface ageing results in interfacial rheological properties consistent with those obtained by drying depending on $\mathrm{WPI} \%_{\mathrm{R}}$. Therefore, we explored the time dependence of the interfacial rheological properties of WPI/NPC dispersions by an oscillatory drop tensiometer.

As already mentioned in the Materials and Methods section, oscillatory experiments were performed once the surface tension equilibrium was reached. Small sinusoidal variations of the mean drop volume are applied to scan the viscoelastic nature of the interfacial layer. Such measurements reveal surface protein self-arrangement as the interface ages, gradually forming complex gelled layers [55]. To reproduce the instantaneous compression rates found in the early stages of the pendant droplet experiments (i.e. Stage 1 in Figure 1), for the oscillatory experiments we applied an amplitude of area deformation amplitude of $10 \%$ and a time period of 30s. In Figure 5, we report the evolution of the complex modulus $E$ with time for WPI/NPC droplets with different WPI $\%_{\mathrm{R}}$. At the beginning $(\mathrm{t}<200 \mathrm{~s})$, samples did not exhibit any viscoelasticity $\left(E^{I}=E^{I I}=1-5 \mathrm{mN} / \mathrm{m}\right)$, except for $\mathrm{WPI} \%_{\mathrm{R}} \geq 80 \%$. With time, proteins re-organized and entangled at drop surface and all samples underwent a sol-gel transition, as underlined by the significant increase of $E$ (up to 5-6 times higher than the initial value when $\mathrm{t}=1600 \mathrm{~s}$ ) and the predominance of the elastic contribution on the viscous one. However, our outcomes also highlighted that both occurrence and importance of gelation significantly depend on WPI $\%_{\mathrm{R}}$. Indeed, the NPC curve $\left(\mathrm{WPI} \%_{\mathrm{R}}=0 \%\right)$ showed to be shifted compared to all others. Samples with $\mathrm{WPI}_{\mathrm{R}} \geq 50 \%$ exhibited a very similar behavior, thus suggesting that WPI colloids control the interfacial rheological properties above a critical concentration in the initial dispersion. It is also worth stressing the surprising behavior observed for $\mathrm{WPI}_{\mathrm{R}}=20 \%$, since $E$ was initially comparable to pure NPC and then increased to higher values than WPI during the measurements. When $t>1600$ s, the $E$ values increased rapidly due to the onset of surface solidification. The occurrence of these events, which implicate microfracture formation and/or other solid-like irreversible deformations, marked the end of the oscillatory tests. Clearly, these outcomes show significant similarities with those obtained by single droplet drying approach. Thus, in interfacial gelation induced by ageing, as for the gelation induced by drying, WPI colloids dominate skin mechanical properties once their concentration is above a same critical value $\left(\mathrm{WPI} \%_{\mathrm{Rc}}\right.$ ). Also, dispersions with WPI $\%_{\mathrm{R}}=20 \%$ confirmed to have unexpected properties, displaying enhanced mechanical response plausibly due to a non-well-defined surface self-arrangement.

\section{Conclusions}

In this work, we provided a characterization of the drying process in droplets of a binary colloidal system consisting of whey proteins (WPI) and casein micelles (NPC). Compared to most of the previous studies focusing on model colloids, here we dealt with two proteins exhibiting significant differences in terms not only of size, but also of structure and mechanical properties. 
The evaluation of droplet profile evolution and drying kinetics with time underlined the crucial role played by WPI in the evaporation dynamics despite being the minor component of the suspension in terms of volume fraction. Indeed, when sufficiently represented in the initial dispersions (WPI $\%_{\mathrm{R}} \geq \mathrm{WPI} \%_{\mathrm{Rc}}$ ), WPI colloids confer to droplets a rigid skin with high resistance to buckling. Such enhanced mechanical properties suggested possible drying-induced WPI external segregation, as already proposed in previous works on bidisperse non-dairy suspensions. The hypothesis of interfacial protein stratification was confirmed by the direct observation of WPI/NPC droplet skin section by SEM, highlighting WPI accumulation on top surface (small-on-top re-arrangement). The occurrence of WPI preferential segregation under certain conditions was interpreted in the light of the ZJD model for binary systems. According to this theory, the accumulation of colloids at droplet surface foster increasing osmotic pressures, which in turn favor the accumulation of smaller macromolecules at the air-liquid interface. Interestingly, although WPI and NPC exhibit different structure and physico-chemical properties that could influence the evaporation process, the size of the two proteins was the main factor governing the auto-stratification. We also showed by oscillatory drop tensiometer that the small-on-top re-arrangement can be also driven by ageing surface gelation. This suggests that the evaporation is not a prerequisite for colloid auto-stratification, but rather the development of local concentration gradients in proximity to droplet surface.

This study represents a point of novelty in the literature about the evaporation in bidisperse colloidal dispersions, since the mechanisms of auto-stratification have been rarely investigated in biological systems so far. Our outcomes corroborate the most accredited theories about drying-induced segregation by size. Moreover, this work represents a forerunner for further investigations that could favor the prediction of dry droplet shape starting basically from colloidal properties (e.g. size, structure, mechanical properties, and surface charge) and composition in a polydisperse dispersion. This could be fundamental, for example, in dairy industry, mostly concerning the optimization of dairy powder production and the control of their functional properties (e.g. rehydration).

\section{Acknowledgments}

This work is supported by the China Scholarship Council (CSC) and Institut Agro / INRAE, in the frame of the FoodPRINT International Associated Laboratory between STLO and the School of Chemical and Environmental of Soochow University.

\section{Bibliography}

[1] C.-C. Peng, C. Cerretani, R.J. Braun, C.J. Radke, Evaporation-driven instability of the precorneal tear film, Adv. Colloid Interface Sci. 206 (2014) 250-264. https://doi.org/10.1016/j.cis.2013.06.001.

[2] L.V. Bel'skaya, E.A. Sarf, A.P. Solonenko, Morphology of Dried Drop Patterns of Saliva from a Healthy Individual Depending on the Dynamics of Its Surface Tension, Surfaces 2.2 (2019) 395-414.

[3] T. Kokalj, H. Cho, M. Jenko, L.P. Lee, Biologically inspired porous cooling membrane using arrayed-droplets evaporation, Appl. Phys. Lett. $96 \quad$ (2010) 163703. https://doi.org/10.1063/1.3332398.

[4] H.M. Gorr, J.M. Zueger, D.R. McAdams, J.A. Barnard, Salt-induced pattern formation in evaporating droplets of lysozyme solutions, Colloids Surf. B Biointerfaces. 103 (2013) 59-66. https://doi.org/10.1016/j.colsurfb.2012.09.043.

[5] J.Y. Kim, B.M. Weon, Evaporation of strong coffee drops, Appl. Phys. Lett. 113 (2018) 183704. https://doi.org/10.1063/1.5049606.

[6] Á.G. Marín, H. Gelderblom, D. Lohse, J.H. Snoeijer, Rush-hour in evaporating coffee drops, Phys. Fluids. 23 (2011) 091111. https://doi.org/10.1063/1.3640018. 
[7] S.J. Temple, A.J.B. van Boxtel, Thin Layer Drying of Black Tea, J. Agric. Eng. Res. 74 (1999) 167176. https://doi.org/10.1006/jaer.1999.0448.

[8] T. Okubo, Sedimentation and drying dissipative structures of green tea, Colloid Polym. Sci. 285 (2006) 331-337. https://doi.org/10.1007/s00396-006-1576-6.

[9] Y.Y. Tarasevich, D.M. Pravoslavnova, Segregation in desiccated sessile drops of biological fluids, Eur. Phys. J. E. 22 (2007) 311-314. https://doi.org/10.1140/epje/e2007-00037-6.

[10] L. Lanotte, D. Laux, B. Charlot, M. Abkarian, Role of red cells and plasma composition on blood sessile droplet evaporation, Phys. Rev. E. $96 \quad$ (2017) 053114. https://doi.org/10.1103/PhysRevE.96.053114.

[11] T.A. Yakhno, A.G. Sanin, V.G. Yakhno, A.S. Pelyushenko, M.B. Dowell, C. Vacca, V.A. Goutorova, A. Analytics, Drying drops of biological liquids: dynamics of the optical and mechanical properties. Application in rapid medical diagnostics, Advanced Biomedical and Clinical Diagnostic Systems III. 5692(2005). https://doi.org/10.1117/12.591556.

[12] J. Park, D. Lee, W. Kim, S. Horiike, T. Nishimoto, S.H. Lee, C.H. Ahn, Fully Packed Capillary Electrochromatographic Microchip with Self-Assembly Colloidal Silica Beads, Anal. Chem. 79 (2007) 3214-3219. https://doi.org/10.1021/ac061714g.

[13] P. Bacchin, D. Brutin, A. Davaille, E. Di Giuseppe, X.D. Chen, I. Gergianakis, F. GiorgiuttiDauphiné, L. Goehring, Y. Hallez, R. Heyd, R. Jeantet, C. Le Floch-Fouéré, M. Meireles, E. Mittelstaedt, C. Nicloux, L. Pauchard, M.-L. Saboungi, Drying colloidal systems: Laboratory models for a wide range of applications, Eur. Phys. J. E. 41 (2018) 94. https://doi.org/10.1140/epje/i2018-11712-x.

[14] D. Bugakova, V. Slabov, E. Sergeeva, M. Zhukov, A.V. Vinogradov, Comprehensive characterization of $\mathrm{TiO} 2$ inks and their application for inkjet printing of microstructures, Colloids Surf. Physicochem. Eng. Asp. 586 (2020) 124146. https://doi.org/10.1016/j.colsurfa.2019.124146.

[15] S. Koga, S. Inasawa, Packing structures and formation of cracks in particulate films obtained by drying colloid-polymer suspensions, Colloids Surf. Physicochem. Eng. Asp. 563 (2019) 95-101. https://doi.org/10.1016/j.colsurfa.2018.11.066.

[16] R.D. Deegan, O. Bakajin, T.F. Dupont, G. Huber, S.R. Nagel, T.A. Witten, Contact line deposits in an evaporating drop, Phys. Rev. E. 62 (2000) 756-765. https://doi.org/10.1103/PhysRevE.62.756.

[17] L. Pauchard, F. Parisse, C. Allain, Influence of salt content on crack patterns formed through colloidal suspension desiccation, Phys. Rev. E. 59 (1999) 3737-3740. https://doi.org/10.1103/PhysRevE.59.3737.

[18] D. Zang, S. Tarafdar, Y.Yu. Tarasevich, M. Dutta Choudhury, T. Dutta, Evaporation of a Droplet: From physics to applications, Phys. Rep. 804 (2019) 1-56. https://doi.org/10.1016/j.physrep.2019.01.008.

[19] A.F. Routh, Drying of thin colloidal films, Rep. Prog. Phys. 76 (2013) 046603. https://doi.org/10.1088/0034-4885/76/4/046603.

[20] G. Gauthier, V. Lazarus, L. Pauchard, Alternating Crack Propagation during Directional Drying, Langmuir. 23 (2007) 4715-4718. https://doi.org/10.1021/la063702w.

[21] E.R. Dufresne, D.J. Stark, N.A. Greenblatt, J.X. Cheng, J.W. Hutchinson, L. Mahadevan, D.A. Weitz, Dynamics of Fracture in Drying Suspensions, Langmuir 22.17 (2006) 7144-7147.

[22] C. Allain, L. Limat, Regular Patterns of Cracks Formed by Directional Drying of a Collodial Suspension, Phys. Rev. Lett. 74 (1995) 2981-2984. https://doi.org/10.1103/PhysRevLett.74.2981.

[23] L. Pauchard, C. Allain, Mechanical instability induced by complex liquid desiccation, Comptes Rendus Phys. 4 (2003) 231-239. https://doi.org/10.1016/S1631-0705(03)00027-6.

[24] A. Fortini, I. Martín-Fabiani, J.L. De La Haye, P.-Y. Dugas, M. Lansalot, F. D’ Agosto, E. BourgeatLami, J.L. Keddie, R.P. Sear, Dynamic stratification in drying films of colloidal mixtures, Phys. Rev. Lett. 116 (2016) 118301. https://doi.org/10.1103/PhysRevLett.116.118301.

[25] C. Le Floch-Fouéré, L. Lanotte, R. Jeantet, L. Pauchard, The solute mechanical properties impact on the drying of dairy and model colloidal systems, Soft Matter. 15 (2019) 6190-6199. https://doi.org/10.1039/C9SM00373H. 
[26] D. Brutin, B. Sobac, C. Nicloux, Influence of Substrate Nature on the Evaporation of a Sessile Drop of Blood, J. Heat Transf. 134 (2012) 061101. https://doi.org/10.1115/1.4006033.

[27] C. Sadek, H. Tabuteau, P. Schuck, Y. Fallourd, N. Pradeau, C. Le Floch-Fouéré, R. Jeantet, Shape, Shell, and Vacuole Formation during the Drying of a Single Concentrated Whey Protein Droplet, Langmuir. 29 (2013) 15606-15613. https://doi.org/10.1021/la404108v.

[28] B. Sobac, D. Brutin, Desiccation of a sessile drop of blood: Cracks, folds formation and delamination, Colloids Surf. Physicochem. Eng. Asp. 448 (2014) 34-44. https://doi.org/10.1016/j.colsurfa.2014.01.076.

[29] L. Pauchard, B. Abou, K. Sekimoto, Influence of nanoparticles mechanical properties on macrocracks formation, Langmuir, 25 (2009) 6672-6677.

[30] A.L.R. Sibrant, L. Pauchard, Effect of the particle interactions on the structuration and mechanical strength of particulate materials, EPL (Europhysics Letters) 116.4 (2017) 49002.

[31] M. Schulz, J.L. Keddie, A critical and quantitative review of the stratification of particles during the drying of colloidal films, Soft Matter. 14 (2018) 6181-6197. https://doi.org/10.1039/C8SM01025K.

[32] R.E. Trueman, Auto-stratification in drying colloidal dispersions: A diffusive model, J. Colloid Interface Sci. 377.1 (2012) 207-212.

[33] R.E. Trueman, E. Lago Domingues, S.N. Emmett, M.W. Murray, J.L. Keddie, A.F. Routh, Autostratification in Drying Colloidal Dispersions: Experimental Investigations, Langmuir. 28 (2012) 3420-3428. https://doi.org/10.1021/la203975b.

[34] R.P. Sear, P.B. Warren, Diffusiophoresis in nonadsorbing polymer solutions: The Asakura-Oosawa model and stratification in drying films, Phys. Rev. E. $96 \quad$ (2017) 062602. https://doi.org/10.1103/PhysRevE.96.062602.

[35] D.K. Makepeace, A. Fortini, A. Markov, P. Locatelli, C. Lindsay, S. Moorhouse, R. Lind, R.P. Sear, J.L. Keddie, Stratification in binary colloidal polymer films: experiment and simulations, Soft Matter. 13 (2017) 6969-6980. https://doi.org/10.1039/C7SM01267E.

[36] M.P. Howard, A. Nikoubashman, A.Z. Panagiotopoulos, Stratification in Drying Polymer-Polymer and Colloid-Polymer Mixtures, Langmuir 33.42 (2017) 11390-11398.

[37] V. Rastogi, S. Melle, O.G. Calderón, A.A. García, M. Marquez, O.D. Velev, Synthesis of LightDiffracting Assemblies from Microspheres and Nanoparticles in Droplets on a Superhydrophobic Surface, Adv. Mater. 20 (2008) 4263-4268. https://doi.org/10.1002/adma.200703008.

[38] L.T. Raju, S. Chakraborty, B. Pathak, S. Basu, Controlling Self-Assembly and Topology at MicroNano Length Scales Using a Contact-Free Mixed Nanocolloid Droplet Architecture, Langmuir. 34 (2018) 5323-5333. https://doi.org/10.1021/acs.langmuir.8b00790.

[39] W. Liu, J. Midya, M. Kappl, H.-J. Butt, A. Nikoubashman, Segregation in Drying Binary Colloidal Droplets, ACS Nano. 13 (2019) 4972-4979. https://doi.org/10.1021/acsnano.9b00459.

[40] R.E. Trueman, E. Lago Domingues, S.N. Emmett, M.W. Murray, J.L. Keddie, A.F. Routh, Autostratification in Drying Colloidal Dispersions: Experimental Investigations, Langmuir. 28 (2012) 3420-3428. https://doi.org/10.1021/la203975b.

[41] J. Zhou, Y. Jiang, M. Doi, Cross-interaction drives stratification in drying film of binary colloidal mixtures, Phys. Rev. Lett. 118 (2017) 108002. https://doi.org/10.1103/PhysRevLett.118.108002.

[42] R.P. Sear, P.B. Warren, Diffusiophoresis in nonadsorbing polymer solutions: The Asakura-Oosawa model and stratification in drying films, Phys. Rev. E. $96 \quad$ (2017) 062602. https://doi.org/10.1103/PhysRevE.96.062602.

[43] Y.F. Yano, Kinetics of protein unfolding at interfaces, J. Phys. Condens. Matter. 24 (2012) 503101. https://doi.org/10.1088/0953-8984/24/50/503101.

[44] A. Bouchoux, G. Gésan-Guiziou, J. Pérez, B. Cabane, How to Squeeze a Sponge: Casein Micelles under Osmotic Stress, a SAXS Study, Biophys. J. 99 (2010) 3754-3762. https://doi.org/10.1016/j.bpj.2010.10.019.

[45] C. Sadek, P. Schuck, Y. Fallourd, N. Pradeau, R. Jeantet, C. Le Floch-Fouéré, Buckling and collapse during drying of a single aqueous dispersion of casein micelle droplet, Food Hydrocoll. 52 (2016) 161-166. https://doi.org/10.1016/j.foodhyd.2015.06.016. 
[46] C. Sadek, L. Pauchard, P. Schuck, Y. Fallourd, N. Pradeau, C. Le Floch-Fouéré, R. Jeantet, Mechanical properties of milk protein skin layers after drying: Understanding the mechanisms of particle formation from whey protein isolate and native phosphocaseinate, Food Hydrocoll. 48 (2015) 8-16. https://doi.org/10.1016/j.foodhyd.2015.01.014.

[47] L. Lanotte, F. Boissel, P. Schuck, R. Jeantet, C. Le Floch-Fouéré, Drying-induced mechanisms of skin formation in mixtures of high protein dairy powders, Colloids Surf. Physicochem. Eng. Asp. 553 (2018) 20-27. https://doi.org/10.1016/j.colsurfa.2018.05.020.

[48] R.G. Picknett, R. Bexon, The evaporation of sessile or pendant drops in still air, J. Colloid Interface Sci. 61 (1977) 336-350. https://doi.org/10.1016/0021-9797(77)90396-4.

[49] E. Mylonas, D.I. Svergun, Accuracy of molecular mass determination of proteins in solution by small-angle X-ray scattering, J. Appl. Crystallogr. $40 \quad$ (2007) s245-s249. https://doi.org/10.1107/S002188980700252X.

[50] A. Bouchoux, P.-E. Cayemitte, J. Jardin, G. Gésan-Guiziou, B. Cabane, Casein Micelle Dispersions under Osmotic Stress, Biophys. J. 96 (2009) 693-706. https://doi.org/10.1016/j.bpj.2008.10.006.

[51] A. Bouchoux, B. Debbou, G. Gésan-Guiziou, M.-H. Famelart, J.-L. Doublier, B. Cabane, Rheology and phase behavior of dense casein micelle dispersions, J. Chem. Phys. 131 (2009) 165106. https://doi.org/10.1063/1.3245956.

[52] R.D. Deegan, O. Bakajin, T.F. Dupont, G. Huber, S.R. Nagel, T.A. Witten, Capillary flow as the cause of ring stains from dried liquid drops, Nature. 389 (1997) 827-829. https://doi.org/10.1038/39827.

[53] D.A. Head, Modeling the elastic deformation of polymer crusts formed by sessile droplet evaporation, Phys. Rev. E. 74 (2006) 021601. https://doi.org/10.1103/PhysRevE.74.021601.

[54] J.S. Nunes, S.J. Bohórquez, M. Meeuwisse, D. Mestach, J.M. Asua, Efficient strategy for hard nanosphere usage: Boosting the performance of waterborne coatings, Prog. Org. Coat. 77 (2014) 15231530. https://doi.org/10.1016/j.porgcoat.2013.11.030.

[55] A. Audebert, A. Saint-Jalmes, S. Beaufils, V. Lechevalier, C. Le Floch-Fouéré, S. Cox, N. Leconte, S. Pezennec, Interfacial properties, film dynamics and bulk rheology: A multi-scale approach to dairy protein foams, J. Colloid Interface Sci. $542 \quad$ (2019) 222-232. https://doi.org/10.1016/j.jcis.2019.02.006. 


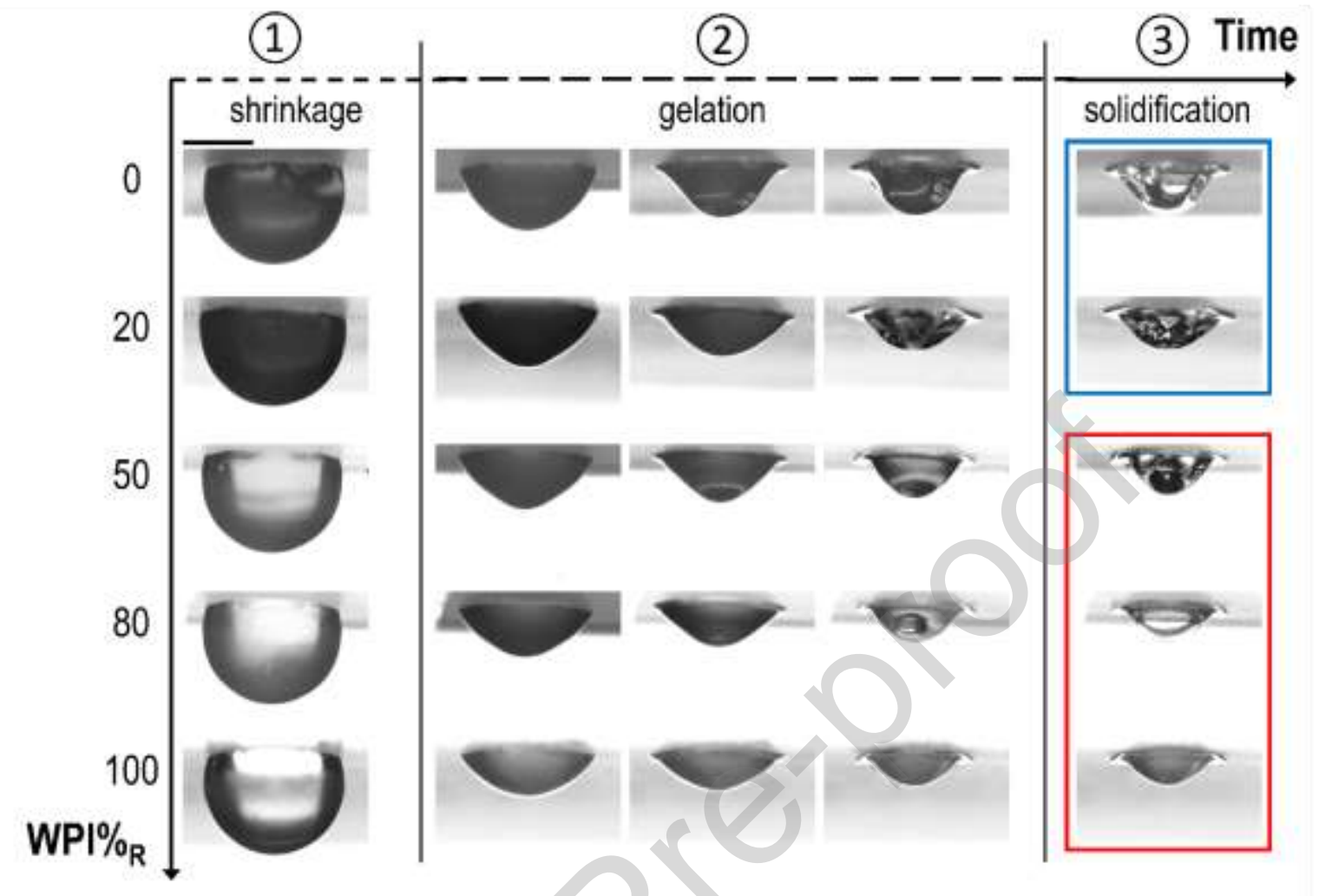

Figure 1: Morphological evolution of WPI/NPC droplets during the evaporation process as a function of WPI\% $\%_{\mathrm{R}}$. The image sequences are intended only to summarize the main drying-induced shape changes, without providing a quantitative comparison between the duration of the different stages. The scale bar is equal to $500 \mu \mathrm{m}$. 


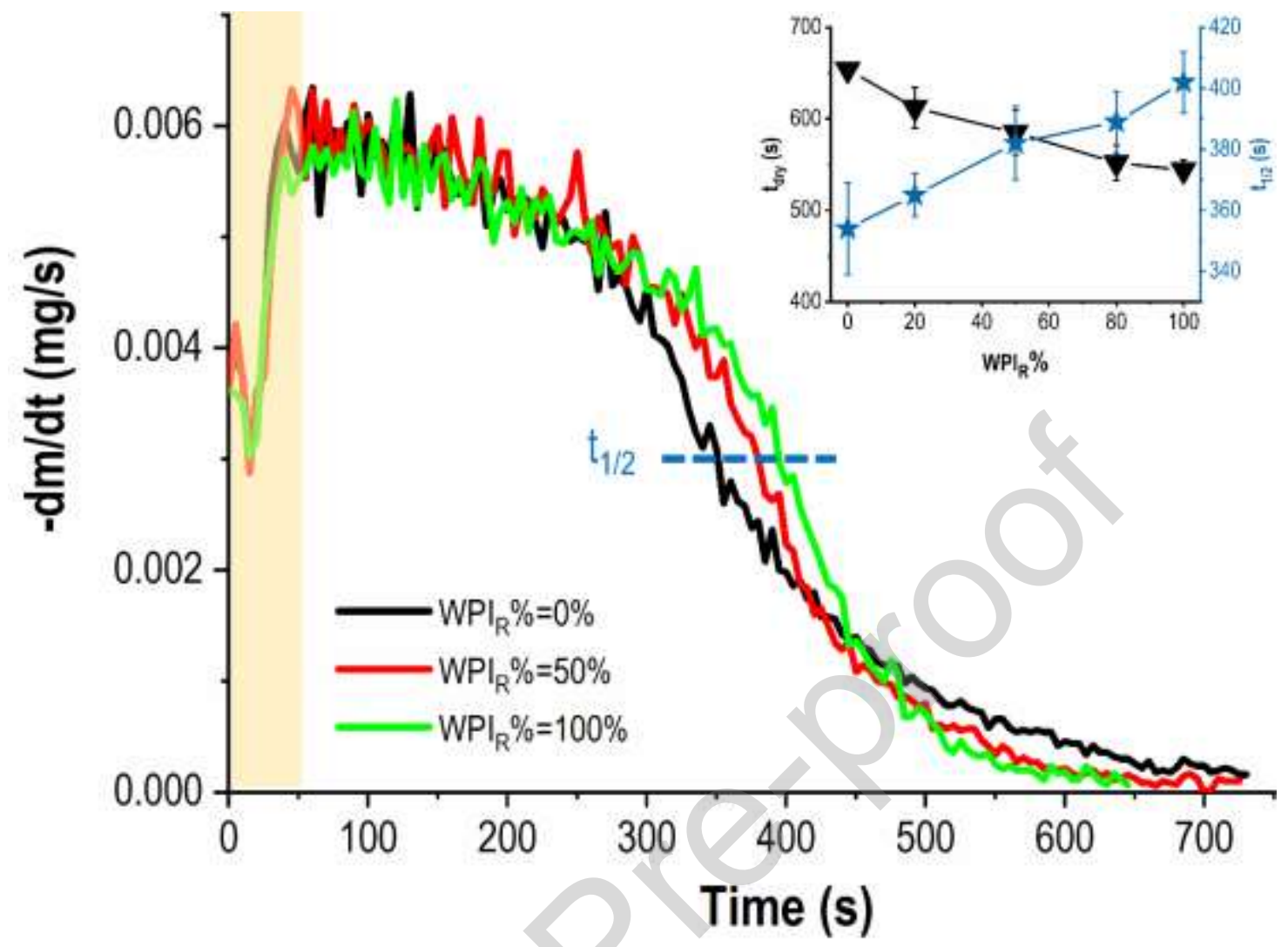

Figure 2: Evolution of the mass loss (-dm/dt) with time in WPI/NPC droplets with WPI\% $=0 \%, 50 \%$, and $100 \%$. In the inset, the black triangles refer to the overall drying time $\left(t_{d r y}\right)$ and the blue stars to the half-life drying time $\left(t_{1 / 2}\right)$ for all the mixes. 


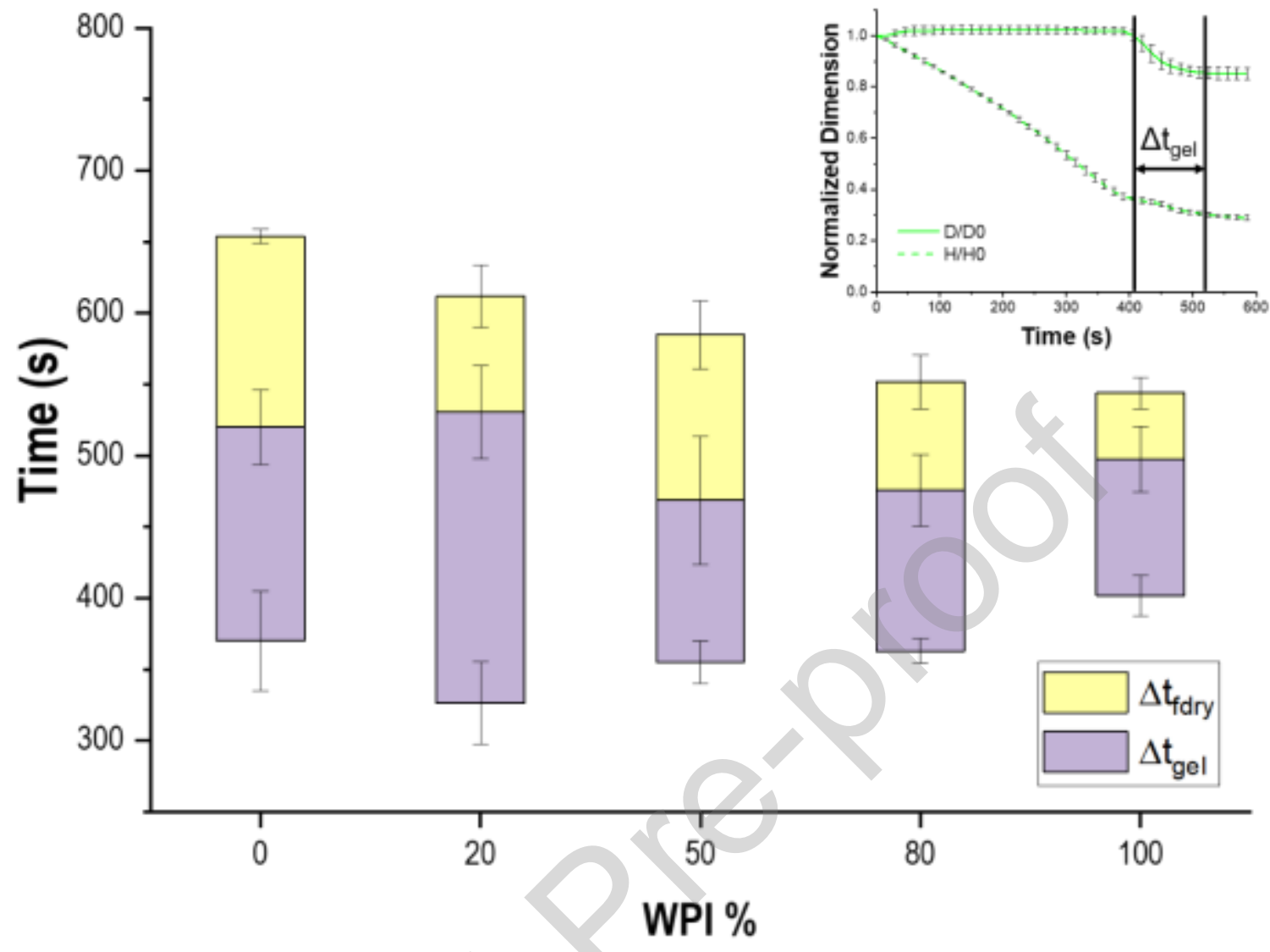

Figure 3: Onset and duration of the buckling stage ( $\Delta t_{\text {gel }}$, purple rectangles), and of the final drying of remaining water $\left(\Delta \mathrm{t}_{\text {fdry }}\right.$, yellow rectangles) as a function of WPI\% $\%_{\mathrm{R}}$. In the inset, typical curves referring to base diameter (D) and apex height $(\mathrm{H})$ time evolution is presented for WPI suspensions. From these curves, the value of $\Delta \mathrm{t}_{\text {gel }}$ has been deduced for all WPI/NPC mixes. 


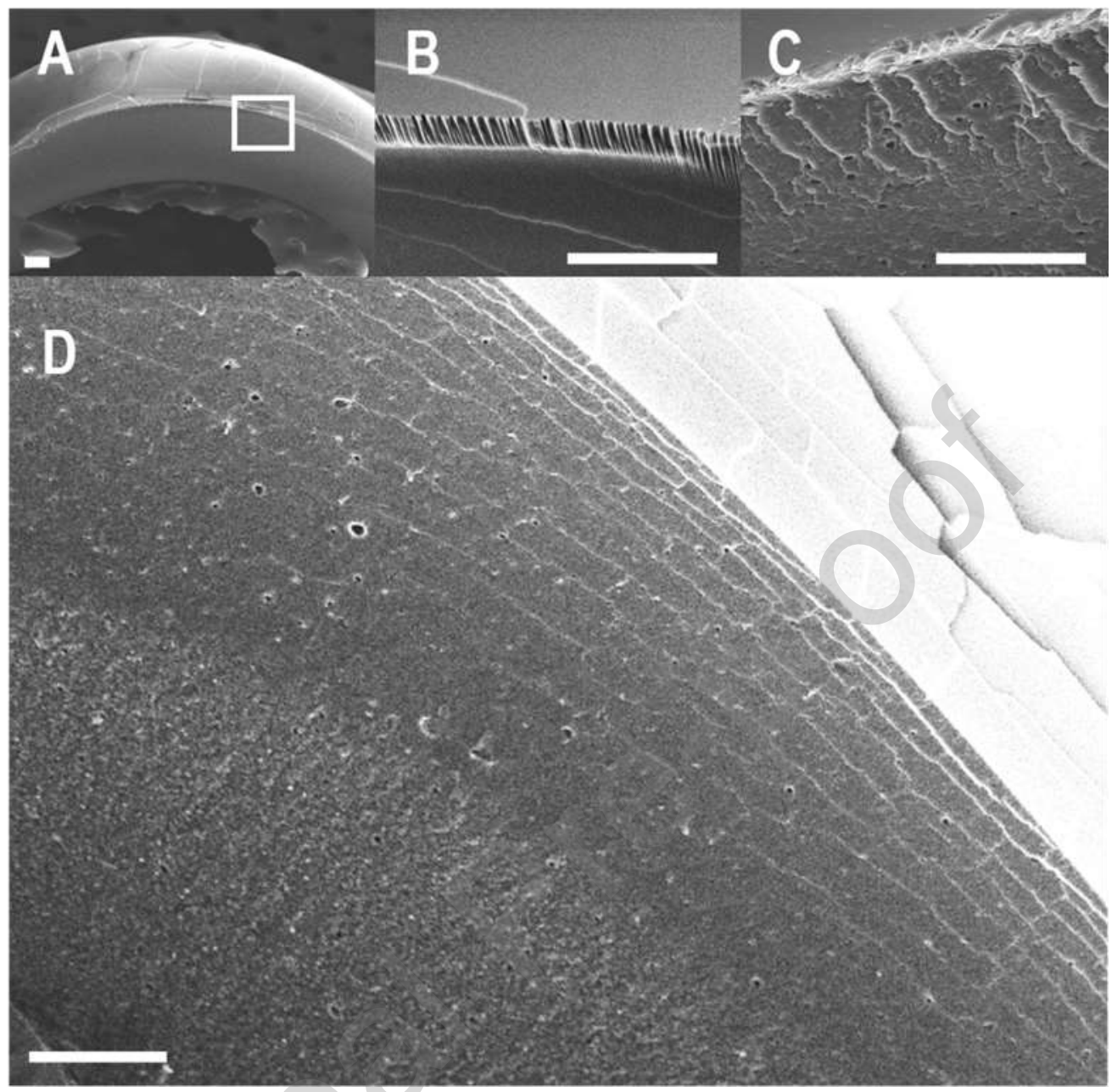

Figure 4: Particle shell structure captured by SEM: A) Skin layer of a dry droplet. The white frame highlights the region of interest for the investigation of skin structure; Shell structure of droplets of WPI (B), NPC (C), and mixes with $\mathrm{WPI} \% \mathrm{R}=\mathbf{5 0 \%}$ (D). The scale bars correspond to $10 \mu \mathrm{m}$. 


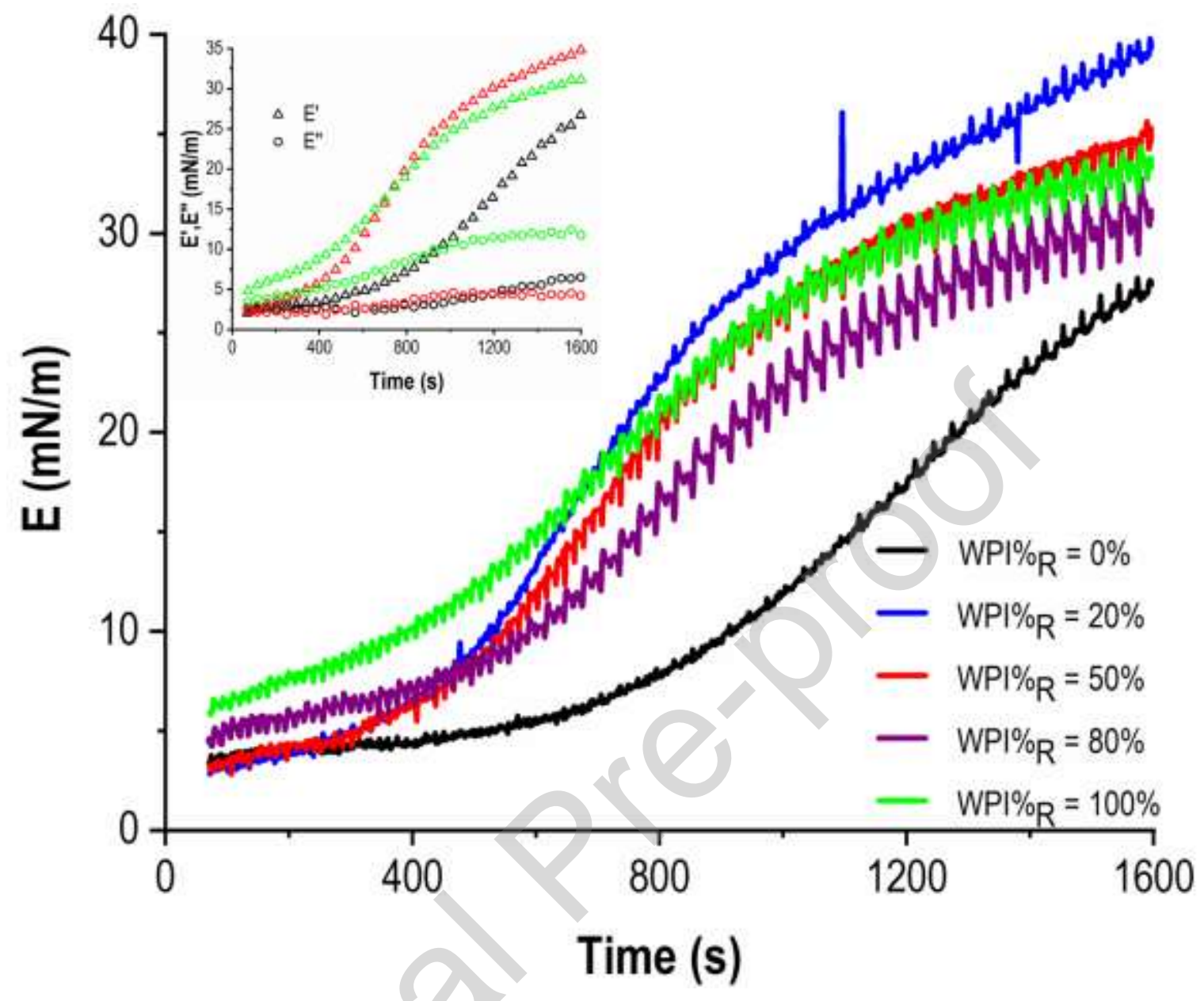

Figure 5: Evolution of the complex viscoelastic modulus $(E)$ with time in WPI/NPC droplets with different WPI\% $\%_{\mathrm{R}}$. In the inset, the elastic $\left(E^{I}\right)$ and the viscous part $\left(E^{I I}\right)$ of the complex modulus are presented for WPI\% ${ }_{\mathrm{R}}=0 \%, 50 \%$, and $100 \%$. 


\section{CRediT author statement}

Ming Yu: Investigation, Formal analysis, Visualization, Writing - Original draft

Cécile Le Floch-Fouéré: Conceptualization, Validation, Supervision

Ludovic Pauchard: Conceptualization, Validation

Françoise Boissel: Investigation

Nan Fu: Conceptualization

Xiao Dong Cheng: Conceptualization, Validation, Supervision

Arnaud Saint-Jalmes: Conceptualization, Investigation, Validation, Writing - Original draft

Romain Jeantet: Conceptualization, Validation, Writing - Reviewing \& Editing, Supervision, Project administration, Funding acquisition

Luca Lanotte: Conceptualization, Investigation, Formal analysis, Validation, Writing - Reviewing \& Editing, Supervision 


\section{Declaration of interests}

$\bigotimes$ The authors declare that they have no known competing financial interests or personal relationships that could have appeared to influence the work reported in this paper.

$\square$ The authors declare the following financial interests/personal relationships which may be considered as potential competing interests:

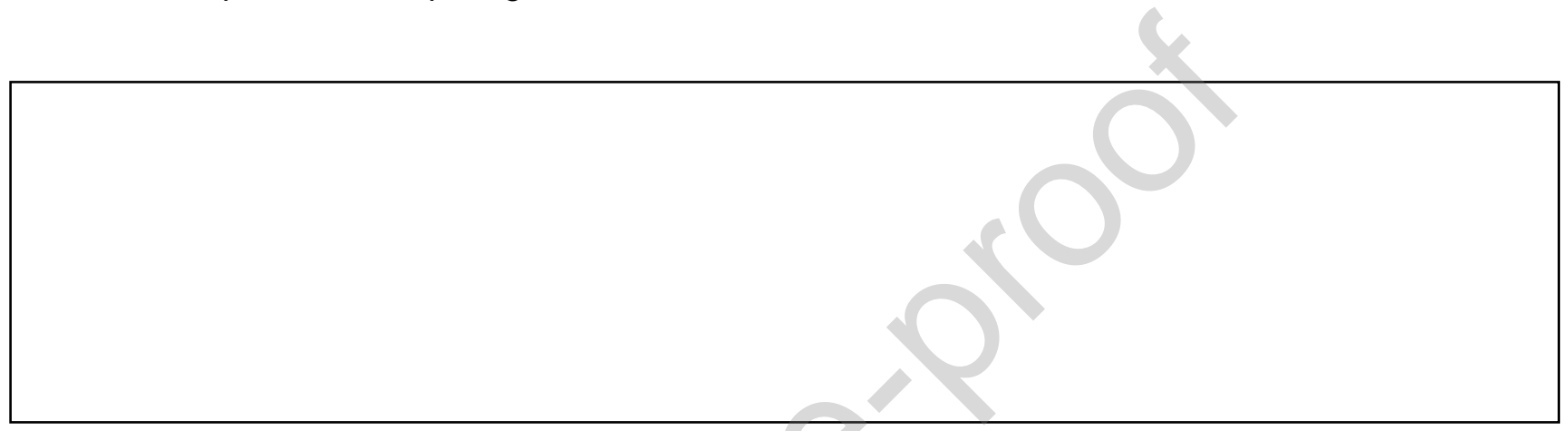

